\title{
To plan or not to plan: Does planning for production remove facilitation from associative priming?
}

\author{
Suzanne R. Jongman ${ }^{\mathrm{a}, *}$, Antje S. Meyer ${ }^{\mathrm{a}, \mathrm{b}}$ \\ a Max Planck Institute for Psycholinguistics, Nijmegen, The Netherlands \\ b Donders Institute for Brain, Cognition and Behaviour, Radboud University, Nijmegen, The Netherlands
}

\section{A R T I C L E I N F O}

\section{Keywords:}

Conversation

Comprehension

Production

Attention

Priming

Picture naming

\begin{abstract}
A B S T R A C T
Theories of conversation propose that in order to have smooth transitions from one turn to the next, speakers already plan their response while listening to their interlocutor. Moreover, it has been argued that speakers align their linguistic representations (i.e. prime each other), thereby reducing the processing costs associated with concurrent listening and speaking. In two experiments, we assessed how identity and associative priming from spoken words onto picture naming were affected by a concurrent speech planning task. In a baseline (no name) condition, participants heard prime words that were identical, associatively related, or unrelated to target pictures presented two seconds after prime onset. Each prime was accompanied by a non-target picture and followed by its recorded name. The participant did not name the non-target picture. In the plan condition, the participants first named the non-target picture, instead of listening to the recording, and then the target. In Experiment 1, where the plan- and no-plan conditions were tested between participants, priming effects of equal strength were found in the plan and no-plan condition. In Experiment 2, where the two conditions were tested within participants, the identity priming effect was maintained, but the associative priming effect was only seen in the no-plan but not in the plan condition. In this experiment, participant had to decide at the onset of each trial whether or not to name the non-target picture, rendering the task more complex than in Experiment 1. These decision processes may have interfered with the processing of the primes. Thus, associative priming can take place during speech planning, but only if the cognitive load is not too high.
\end{abstract}

\section{Introduction}

When we speak, we most often do this in conversation, in alternation with someone else. Unless we are presenting at a conference or teaching a class, we expect that the people we are talking to will reply. A conversation implies that two or more people are actively contributing to the discussion. A conversation also implies that each individual not only speaks, but also listens. In other words, the processes of language production and language comprehension must be combined when one takes part in a conversation. Yet, language production and language comprehension have been investigated largely separately. Surprisingly little is known about what happens to these two fundamental components of language in coordination with one another, even though this is the natural way these two processes occur in our daily lives.

Results of corpus studies suggest that production and comprehension can be readily combined with each other. A consistent finding across many languages is that gaps between speakers' turns are often as short as 200 ms (Heldner \& Edlund, 2010; Stivers et al., 2009). In other words, after listening to someone else's speech, one's own response is already initiated within $1 / 5$ of a second. This is surprising because studies that have investigated language production in isolation have shown that speakers need on average around $600 \mathrm{~ms}$ to plan a single object name (Indefrey \& Levelt, 2004) and at least a second to produce a description of an action picture in a phrase such as "The dog chases the mailman" (Konopka \& Meyer, 2014). Though substantial parts of these planning times may be taken up by the visual processing of the pictures, and no precise estimates are available about the time speakers need to transform their own thoughts into utterances, these studies strongly suggest that speakers usually need $>200 \mathrm{~ms}$ to plan a turn. The short gaps between turns therefore indicate that utterance planning already starts while one is still listening to the preceding speaker. Levinson and Torreira (2015) proposed that speakers begin to plan their utterances as soon as they have enough information to decide what to say. Specifically, they claimed that words are fully planned all the way to the stage prior to articulation, that this information may be held in working memory until the end of turn is predicted, and that articulation is launched just prior to the end of turn.

\footnotetext{
* Corresponding author at: Max Planck Institute for Psycholinguistics, PO Box 310, 6500 AH Nijmegen, The Netherlands.

E-mail address: suzanne.jongman@mpi.nl (S.R. Jongman).
} 
Recent experimental evidence is consistent with this early planning hypothesis. Bögels, Magyari, and Levinson (2015) designed a quiz EEG study where the answer could either be deduced early in the question or only at the very end (e.g., "Which character, also called 007, appears in the famous movies?" versus "Which character from the famous movies is also called 007?"). Participants were $310 \mathrm{~ms}$ faster to answer in the early than in the late condition. Moreover, in the EEG signal a late positivity starting around $500 \mathrm{~ms}$ after the onset of the critical information was observed for both the early and the late condition. This positivity was reduced in a control condition where participants did not have to answer but only to remember the questions. The authors suggested that the late positivity reflected response planning. These results reveal that participants started planning their answer as soon as they had enough information to do so. Barthel, Sauppe, Levinson, and Meyer (2016) obtained similar evidence using a list-completion paradigm where a participant and a confederate both saw sets of objects on their screens. The confederate named her items first, and the participant then named any additional items they saw on their screen. Importantly, the confederate's description either ended in a noun or a verb form (e.g. "Ich habe eine Puppe und einen Schuh (besorgt) - I have a doll and a shoe (obtained)"). The participants' speech onset latencies were shorter in the verb-final than in the noun-final condition. This indicates that they began to plan their utterances as soon as they had heard the final noun and knew which objects they had to name. These studies show that the processes of comprehension and production can co-occur in time. An important task for theories of language processing is to explain how they are coordinated.

Both language comprehension and production have been shown to be capacity demanding. For instance, Rodd, Johnsrude, and Davis (2010) showed that participants were slower to decide whether visually presented letters were printed in upper or lower case when sentences they heard simultaneously required semantic disambiguation compared to unambiguous sentences. This indicates that meaning selection during comprehension interfered with response selection for the visual task. Similarly, Ferreira and Pashler (2002) showed that increasing the difficulty in a word production task delayed responses in an unrelated concurrent non-linguistic task, indicating that both tasks drew from the same pool of attentional resources (see also Cook \& Meyer, 2008). Individual differences research points in the same direction, for instance by showing that individuals with better working memory abilities perform better in language comprehension (Huettig \& Janse, 2016) and language production tasks (Shao, Roelofs, \& Meyer, 2012) than individuals with weaker memory abilities.

In essence, listening to another person while planning one's own utterance is a dual-task situation and one would expect the two tasks to interfere with each other. This expectation has been confirmed in numerous picture-word interference (PWI) experiments, where participants name pictures while listening to stimuli they should ignore. These experiments have shown that participants are slower to name the pictures in the presence of words than noise or pseudowords (Dhooge \& Hartsuiker, 2012; Schriefers, Meyer, \& Levelt, 1990). Relatedly, Fargier and Laganaro (2016) showed that naming latencies increased when pictures were paired with auditorily presented linguistic stimuli (syllables) than with non-linguistic stimuli (tones) on which participants performed a categorization task. Their findings are consistent with the results of numerous dual-task studies showing that similar tasks interfere more with each other than more dissimilar ones (Navon \& Miller, 1987; Wickens, 2008).

In sum, all of these studies demonstrate that speech planning is hindered by concurrent processing of words. However, the degree of hindrance depends to some extent on the relationship between the perceived and planned words: Compared to unrelated words, categorically related ones yield additional interference in picture-word interference paradigms, whereas associatively related words often yield facilitation (Abdel Rahman \& Melinger, 2007). This pattern is consistent with effects found for priming of picture naming (Alario, Segui, \& Ferrand, 2000; Perea \& Gotor, 1997). Primes that are identical to the picture yield strongest facilitation (Ferrand, Grainger, \& Segui, 1994; Wheeldon \& Monsell, 1992). These findings are important in the current context because successive utterances in conversation are often related, and such relationships may either alleviate or increase the interference that should arise when speech planning and listening cooccur. In fact, Garrod \& Pickering, 2004 have argued that speakers prime each other, thereby making dialogue an easier task than speaking in a monologue. Indeed, conversation partners tend to use the same word to describe an object as their interlocutor even if alternate labels are available (Brennan \& Clark, 1996; Garrod \& Anderson, 1987).

\section{The present study}

Picture-word interference studies and the study by Fargier and Laganaro (2016) investigated how picture naming was affected by concurrent processing of nonverbal and verbal stimuli. By contrast, the present study explored how the processing of spoken words was affected by the presence or absence of a concurrent word planning task. Thus, while it has already been established that speech planning is affected by listening, we investigated how listening is affected by speech planning. If it is both true that speakers prime each other and that speech planning overlaps with listening, it is of theoretical interest to demonstrate that mutual priming can indeed occur during concurrent speech planning. To test this, we used a novel priming paradigm. Participants saw a stream of pictures and named them as quickly as possible. The pictures were preceded, with an SOA of $2 \mathrm{~s}$, by spoken prime words. The primes were identical to the target names, associatively related, or unrelated. We are not aware of picture priming studies using such a long SOA, but given the results of existing picture naming studies (Alario et al., 2000; Ferrand et al., 1994; Perea \& Gotor, 1997) and word processing studies using associative and identity priming (Gomez, Perea, \& Ratcliff, 2013; Moss \& Marslen-Wilson, 1993; Shelton \& Martin, 1992), we expected strong facilitation from both types of related primes. Most likely, these effects are a mixture of automatic processes (Collins \& Loftus, 1975) and strategic processes coming into play with longer SOAs (Bodner \& Masson, 2001; de Groot, 1984).

Our main question was how these priming effects would be affected by a concurrent speech planning task. To assess this issue, we combined each prime with a non-target picture as illustrated in Fig. 1. One group of participants (the no-plan group) did not react overtly to these pictures but heard a recording of the corresponding name $600 \mathrm{~ms}$ after picture onset. Thus, the participants simultaneously saw a non-target picture and heard a prime, then heard a recording of the name of the non-target picture, and finally named the target picture. Note that the name of the non-target picture intervened between the prime and the target picture. However, given the anticipated strength of the priming effects and their largely strategic basis, we expected these effects to be present in spite of the intervening name of the non-target picture.

A second group of participants saw the same pictures and heard the same prime words but had to name the non-target pictures as well as the target pictures. Thus, these participants planned the names of the non-target pictures while hearing the primes. We assessed whether the strength of the priming effects differed between the two groups. One possibility is that speakers can easily process spoken words and use them strategically (i.e. as primes) while preparing their own utterances. If that is the case, similar patterns of results should be seen in the two groups. This pattern would be in line with the proposal in the literature on conversational turn-taking that this kind of dual-tasking is facilitated by mutual priming and as such no costs are associated with speech planning with concurrent listening (Garrod \& Pickering, 2004; Pickering \& Garrod, 2004). Alternatively, in line with literature on dualtask experiments (Navon \& Miller, 1987; Wickens, 2008), speakers may not be able to process spoken words as efficiently when they are planning utterances as when they are just listening to the words. In that case, the priming effects should be absent or much reduced in the plan compared to the no-plan group. 
A

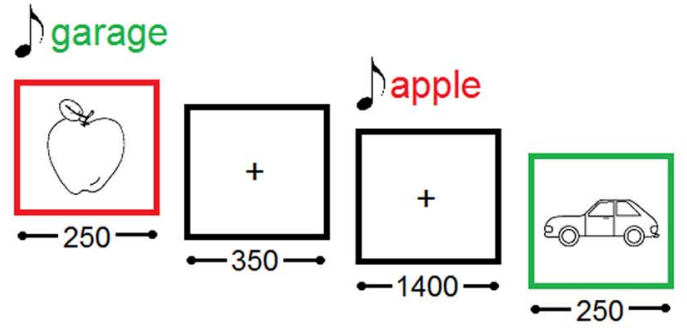

$\mathrm{B}$

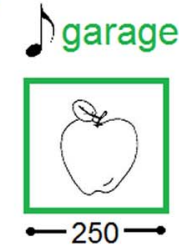

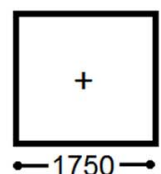
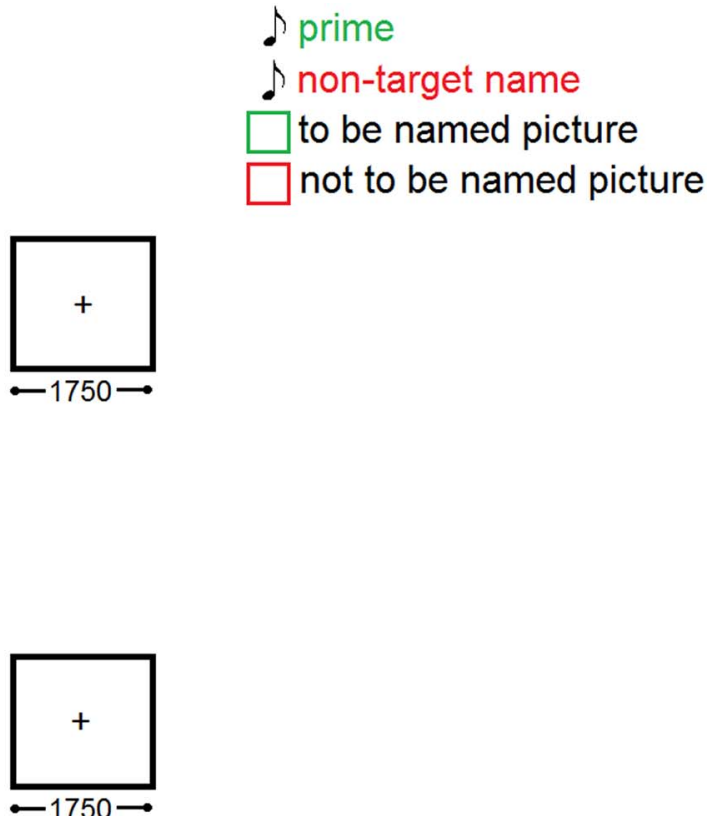



Fig. 1. Stimuli in the no-plan group (panel A) and plan group (panel B). The example depicts a trial from the associatively related condition.

Our novel priming paradigm resembles the picture-word interference task but there are important differences between the paradigms. The first picture (our non-target) is presented together with an auditory stimulus just as in many PWI tasks. Here we refer to the auditory word as the prime, whereas in PWI studies it is known as the distractor, but the combination of a heard word and a picture is identical. However, we are not interested in the effect of this auditory word on the ease of naming the picture shown at the same time, but in its effect on the ease of naming the target picture presented $2 \mathrm{~s}$ later. Therefore an important difference between the two paradigms is the timing of the critical stimuli. Moreover, whereas PWI studies usually concern issues of word production, we are interested in spoken word processing: We examine the picture naming latencies in order to understand how well the preceding spoken prime word was processed. We know from PWI studies that the auditory word impacts naming when the word and picture are presented close in time, and therefore we know that both stimuli are processed even when these processes are forced to take place concurrently. However, this does not imply that processing of the auditory word is unaffected by naming a picture, i.e. the degree of processing could be different when compared to a situation where listening to the auditory word is the sole task.

The paradigm used here is obviously far removed from actual conversation. We rarely only hear one word and subsequently utter just one word. However, this method allows for careful manipulation of what is heard, what is said, and when it is said. In the previous studies investigating the coordination for comprehension and planning for production, participants were free to start planning when they wanted (Barthel et al., 2016; Bögels et al., 2015; Sjerps \& Meyer, 2015). Although measures such as eye-tracking and EEG were used to determine when participants started planning, it remains difficult to pinpoint the exact time when planning started for each trial. Moreover, it is unclear to what level (i.e. to the semantic or phonological level) words were planned initially. In the current paradigm, participants are forced to fully plan an object name while listening. Thus, we know both when and what they planned. This method can be used to investigate different relationships between what is heard and said and can be used to test both issues related to capacity sharing theories of speaking and listening on the one hand and psycholinguistic theories on conversation on the other hand.

\section{Experiment 1}

\subsection{Method}

\subsubsection{Participants}

Thirty-three participants, who were students at Radboud University Nijmegen or the Hogeschool van Arnhem en Nijmegen, participated in the experiment and received a monetary reward. All participants were native speakers of Dutch and had normal or corrected-to-normal vision and audition. Data from one participant was removed, due to a technical error. The average age of the remaining thirty-two participants was 21.1 years (range 18-27 years). Twenty-nine participants were female, and twenty-seven were right-handed. Approval to conduct the study was granted by the Ethics Committee of the Social Sciences Faculty of Radboud University.

\subsubsection{Materials and design}

Forty-eight pictures were selected from a database of normed pictures (Severens, Van Lommel, Ratinckx, \& Hartsuiker, 2005). Pictures were selected to have high name agreement (mean: 98.4\%, range: 90-100). The object names varied in frequency (mean: 107 tokens per million; range: 3-820; CELEX, Baayen, Piepenbrock, \& Gulikers, 1995) and length from one to three syllables (mean: 1.5). Pictures were presented as white line drawings in the middle of a black screen, fit to a virtual frame of 300 by 300 pixels (17 inch Iiyama LM704UT screen).

The auditory primes were identical, associatively related, or unrelated to the targets. For the related and unrelated condition, association norms were retrieved from the Dutch word association project (De Deyne \& Storms, 2008). Associative primes belonged to a different semantic category and were highly associative cues to the targets. The mean number of times the target was given as a response to the prime divided by all responses to the prime was 0.23 (range: 0.13 to 0.31 , see Appendix A for all prime-target combinations and their norms). Associative primes varied in frequency (mean: 13 tokens per million, range: 
0-78) and in length (mean: 1.9 syllables, range: 1 to 4 syllables). The same primes were used in the unrelated condition in combination with different targets to result in associative relatedness scores of 0 for all items. The auditory primes were spoken with neutral intonation by a female speaker. The recorded sound files were normalized such that the intensity was similar for all primes. Auditory primes were presented over headphones (Sony MDR-V150).

We used an SOA of $2 \mathrm{~s}$ so that there would be enough time for the participant to name the non-target picture before the target picture appeared. This SOA is longer than SOAs that have been used previously; it seems $1 \mathrm{~s}$ is the longest SOA in the literature (de Groot, 1984; Keefe \& Neely, 1990). Therefore, a pilot study was conducted with 12 participants to assess whether identity and associative priming would be obtained with this long SOA (see Appendix B for experimental and statistical details). Each prime-target combination was presented twice, once in each half of the experiment. Participants were asked to name the targets. A linear mixed effects model revealed robust priming effects. In the unrelated condition, participants were slowest with an average naming time of $652 \mathrm{~ms}(\mathrm{SD}=168)$. When participants first heard an associatively related prime, they named pictures in $581 \mathrm{~ms}$ ( $\mathrm{SD}=193)$. Finally, participants were quickest in the identity condition: $509 \mathrm{~ms}(\mathrm{SD}=144)$.

In the main experiment, non-target pictures were presented simultaneously with the auditory primes. Target pictures were never accompanied by a sound file. The non-target pictures were taken from the same set of 48 pictures as the target pictures. In the no-plan group the non-target picture were not named by the participant. Instead the name of non-target picture was played $600 \mathrm{~ms}$ after picture onset. Tobe-named pictures were surrounded by a green frame and pictures that did not require a response by a red frame.

Each picture was presented twelve times, six times as the target picture and six times as the non-target picture. The experiment was divided into six blocks of 48 trials. Each picture appeared as a target exactly once in each block, and as a non-target zero to three times. Each prime condition appeared 16 times each block. Across participants, every prime-target combination was presented in each of the blocks the same number of times. Within a block, the presentation of the pictures was pseudorandomized such that two objects of the same semantic category never followed one another, nor did two names starting with the same phoneme. These constraints held both within trials (for the non-target picture and target picture) and between trials (for the target picture of one trial and the non-target picture of the next trial). Similarly, primes were never immediately repeated. Moreover, no more than two trials of the same condition occurred in a row.

\subsubsection{Procedure}

Before the experiment, participants were familiarized with the pictures and their names. Each picture was presented in the middle of the screen with its name written underneath. The participant was asked to study the drawings and their names and use only the proposed names in the experiment. They pressed "Enter" to proceed from one picture to the next. After all 48 pictures had been presented, a short practice block was presented. Three new pictures were introduced and each of them was presented three times. In the no-plan group the pictures were surrounded either with a green frame (to be named picture) or red frame (not to be named picture), whereas in the plan group all pictures were accompanied by a green frame.

A trial started with the simultaneous presentation of a prime word and a non-target picture (see Fig. 1). The non-target picture was presented for $250 \mathrm{~ms}$, followed by a fixation cross in the middle of the screen. The fixation cross remained on the screen for $1750 \mathrm{~ms}$. In the no-plan group, the name of the non-target picture was presented $600 \mathrm{~ms}$ after the onset of the picture. This SOA was chosen to approximate the estimated naming latency of the non-target pictures in the plan-group. Two seconds after trial onset, the second picture, the target, was presented for $250 \mathrm{~ms}$. The trial ended with a fixation cross being presented for $1750 \mathrm{~ms}$. The participants in the plan group were instructed to name all pictures, whereas the participants in the no-plan group were instructed to name only the pictures with green frames.

At the beginning of the experiment and after the end of the break, a fixation cross was presented for $1 \mathrm{~s}$ to draw participants' attention to the screen. Participants received a short break in the middle of the experiment, thus after 144 trials. The experimental session lasted approximately $20 \mathrm{~min}$.

\subsection{Results}

\subsubsection{Data analysis}

Vocal responses were recorded by a microphone (Sennheiser ME64). Naming errors and hesitations were coded online and discarded from the analyses. RTs were determined manually using the program Praat (Boersma \& Weenink, 2012). The naming latencies for correct responses were analyzed using R ( $\mathrm{R}$ Core Team, 2012) and the R packages lme4 (Bates, Maechler, \& Bolker, 2013) and languageR (Baayen, 2011). The latencies were log-transformed due to positive skewing. The linear mixed effects model included condition (identity, related, and unrelated prime) and group (plan and no-plan) as fixed effects, and their interaction. As condition had three levels, contrast coding was chosen such that the related condition was compared to the identity condition, which was expected to yield faster responses, and the unrelated condition, which was expected to yield slower responses. Variables that did not reliably contribute to model fit were dropped. Models were compared using a likelihood ratio test. Participant, target, and prime were treated as random effects. The maximal random effects structure was chosen (Barr, Levy, Scheepers, \& Tily, 2013). For participant the random slope for condition was included, for target both the slopes for condition and group and its interaction. The model provides estimates, standard errors and $t$-values for each coefficient; factors with absolute values of $t>2$ were considered to significantly contribute to explaining the dependent variable (Baayen, 2008).

For the plan group, a second model was run for the first picture that was named. The only fixed effect was condition and its slope was included for the random effects participant and target. No effect was expected, because the non-target pictures were always unrelated to the prime. Finally, we also tested a model where the naming latencies for the first picture and second picture were compared. The only fixed effect was picture position, and its slope was included for the random effects participant and picture. We expected naming latencies to be longer for the first picture than the second picture, as the first was accompanied by the auditory prime whereas the second picture was presented on its own.

\subsubsection{Response times}

Naming errors for the target pictures occurred on average on $0.6 \%$ of all trials, and in an additional $0.9 \%$ of trials participants hesitated. No error analysis was performed due to the low number of mistakes. In the plan group, participants also named the first, non-target picture. Participants gave incorrect responses to non-target pictures on $0.9 \%$ of the trials and hesitation on $0.3 \%$ of the trials. Only trials with correct responses for both pictures were included in the RT analysis.

The picture naming latencies showed strong identity and associative priming effects. As Fig. 2 shows these effects were similar in size in the plan and no-plan condition. The best-fitting linear mixed effects model for correct naming latencies was the model that only included the fixed effect of condition. Removing the interaction from the full model did not result in poorer model fit $\left(\chi^{2}(2)=3.57, p=0.17\right)$. Removing the fixed effect group did not decrease model fit either $\left(\chi^{2}(1)=0.79\right.$, $p=0.37)$. Finally, removing condition did result in a significant result $\left(\chi^{2}(2)=30.69, p<0.001\right)$. Thus, the best fitting model only included condition. The first contrast for conditions, the related versus the identity condition, was significant $(\beta=-0.069, \quad \mathrm{SE}=0.012$, $t=-5.61$, related: $646 \mathrm{~ms}(\mathrm{SD}=210)$ vs. identity $597 \mathrm{~ms}(\mathrm{SD} 169)$ ). 


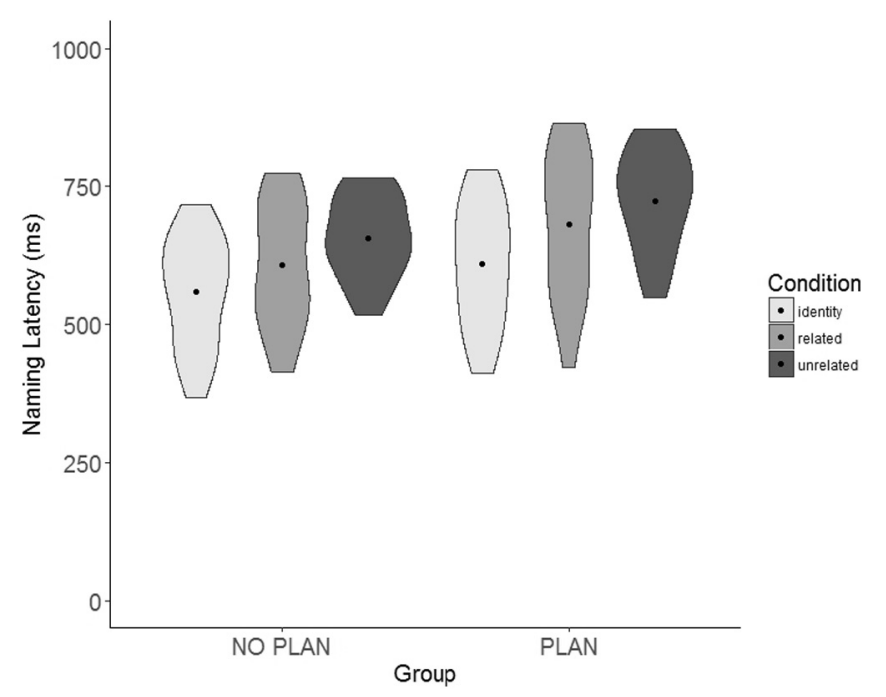

Fig. 2. Violin plot of mean naming latencies for target pictures following either an identity, associatively related or unrelated prime; separated for the no-plan group and plan group. Dots indicate the condition mean.

The second contrast, between the related and unrelated condition, also showed a significant difference $(\beta=0.069, \mathrm{SE}=0.017, t=3.99$, unrelated: $679 \mathrm{~ms}(S \mathrm{~S}=183)$ ). Since naming in the identity condition was significantly faster than in the related condition, and naming in the related condition was significantly faster than in the unrelated condition, we can also conclude that the identity condition was significantly different from the unrelated condition.

In the plan group, participants named the first picture as well. The average naming latency for these first pictures was $813 \mathrm{~ms}(\mathrm{SD}=232)$. A null model was compared to a model with condition as a fixed effect: adding condition did not result in better model fit $\left(\chi^{2}(2)=0.50\right.$, $p=0.78)$. As there was no relationship between the prime and the first picture, there is no effect of condition on the first naming latencies. Finally, naming latencies for the first picture were compared to those for the second picture. We found a significant increase in model fit when adding picture position to the respective null model $\left(\chi^{2}(1)\right.$ $=41.05, p<0.001, \beta=-0.204, \mathrm{SE}=0.015, t=-13.22$ ). In other words, participants were slower to name pictures presented in the first position (non-targets, accompanied by an auditory prime) than in second position (targets, presented without primes).

\subsection{Discussion}

Strong identity and associative priming effects were found in both the no-plan and the plan group. Overall, even though there was an intervening picture between the prime and target, the standard priming pattern was found: fastest naming for pictures preceded by an identity prime, intermediate naming latencies for associatively related primes and slowest naming after unrelated primes. In the pilot study, where participants only heard the primes and named the target pictures, we obtained identity and associative priming effects of $143 \mathrm{~ms}$ and $71 \mathrm{~ms}$, respectively. In the main experiment, these effects were reduced to 82 and $33 \mathrm{~ms}$, respectively, but still highly significant. The reduction of the size of the effects was most likely due to the processing of the accompanying non-target pictures and their names. Importantly, the plan and no-plan groups did not differ in the size of the priming effects. Thus, judging from the size of the priming effects, preparing to name the nontarget pictures did not interfere more with the processing of the spoken prime words than merely inspecting the pictures. This result implies that people can readily combine planning their own utterances with attentively listening to another person's speech.

In Experiment 2 we set out to replicate these results. One goal was to validate the novel paradigm used in Experiment 1 in a study using a different design, switching from between-participants to within-participants variation of the plan/no-plan variable. In Experiment 2, half of the trials were identical to the trials presented to the no-plan group and the other half were identical to the trials presented to the plan group. A second goal of Experiment 2 was to find corroborating evidence that the beneficial effects of priming remained during concurrent speech planning with increased complexity. In Experiment 1, participants in the plan-group named all pictures, and the participants in the no-plan group named every other picture. Thus, all participants could predict with certainty which pictures they would have to name. It is therefore possible that they quickly began to use the primes strategically, as shown in earlier priming experiments using long SOAs (Bodner \& Masson, 2001; de Groot, 1984). In the within-participants design of Experiment 2 the succession of pictures that had to be named, or did not have to be named, was random. It only became clear at the start of the trial, indicated by the color of the frame around the picture, whether or not the picture had to be named. The unpredictability may change the strategic processing of the primes.

\section{Experiment 2}

\subsection{Method}

\subsubsection{Participants}

Thirty-eight new participants were selected from the same participant pool as Experiment 1. Data from two participants were removed, one due to failure to follow task instructions, the other due to frequent mispronunciations. The average age of the remaining 36 participants was 22.2 years (range 19 to 28 years). 29 participants were female, and 28 participants were right-handed.

\subsubsection{Materials and design}

The materials were identical to the previous study. On half of the trials, the first picture of a trial (the non-target picture) was surrounded by a red frame and followed by its name after $600 \mathrm{~ms}$ spoken by a prerecorded speaker as in the no-plan group of Experiment 1 (here called the no-plan condition). On the other half of the trials, both pictures in one trial were surrounded by green frames and both should be named (as in the plan group of Experiment 1, here the plan condition).

\subsubsection{Procedure}

On half of the trials, the procedure was identical to the trials presented in the no-plan group of Experiment 1. On the other half, the procedure was identical to the procedure of the plan group. Trials were pseudorandomized as in Experiment 1, with the addition that no more than five trials in the no-plan or plan condition followed one another. Participants were asked to name all pictures surrounded by a green frame.

\subsection{Results}

\subsubsection{Data analysis}

The fixed effects included in the model for the naming latencies for target pictures were condition (identity, related, or unrelated prime), plan (yes or no) and their interaction. Models were compared using a likelihood ratio test. Using backward elimination the optimal model was chosen. The random structure included the intercept for participant, target and prime condition. Moreover, slopes were included for condition and plan for both participants and targets. The slope for the interaction between condition and plan was included for participant.

A model was run for the first picture that was named, containing only the trials in the plan condition. The only fixed effect was condition and its slope was included for the random effects participant and target. Moreover, we compared naming latencies for the first and second picture of a trial, i.e. for non-target pictures to target pictures. The only fixed effect was picture position and its slope was included for participant and target. 


\subsubsection{Response times}

For the target pictures, participants gave erroneous answers on $0.4 \%$ of the trials and hesitated on another $0.6 \%$ of the trials. These trials were excluded from the following analyses. Moreover, trials where participants had trouble naming the first picture in the plan condition were removed (errors: $1.0 \%$, hesitations: $0.7 \%$ ). Participants had no problem following task instructions and naming only the pictures presented with green frames (error rate $<0.1 \%$ ), and not naming the pictures with red frames (error rate $<0.1 \%$ ). No error analysis was performed due to the small number of mistakes.

The speech onset latencies for target naming in the six experimental conditions are shown in Fig. 2. In both the plan and the no-plan condition, identity primes resulted in strong facilitation. In the no-plan condition associative primes also led to facilitation. This was not the case in the plan condition. Thus, in this experiment the effects of the associative primes were different in the two plan conditions.

The best-fitting linear mixed effects model for correct naming latencies for the target pictures included both main effects and their interaction. Removing the interaction between condition and plan significantly decreased model fit $\left(\chi^{2}(2)=7.64, p<0.05\right)$. To examine the interaction, the data was split by plan condition. The main effect of condition was significant in both plan trials and no-plan trials. In the no-plan trials, condition improved model fit $\left(\chi^{2}(2)=51.88\right.$, $p<0.001)$. The first contrast, comparing the related versus the identity condition, was significant $(\beta=-0.085, \mathrm{SE}=0.012, t=-7.00$, related: $646 \mathrm{~ms}$ (SD 195) vs. identity: $584 \mathrm{~ms}$ (SD 140)). The second contrast, between the related and unrelated condition, also showed a significant difference ( $\beta=0.041, \mathrm{SE}=0.009, t=4.30$, unrelated: 662 (SD 165)). We can also conclude from this pattern of data that the identity condition not only differed from the related condition, but also from the unrelated condition.

In the plan trials, condition also improved model fit $\left(\chi^{2}(2)=52.76\right.$, $p<0.001)$. The first contrast, the related versus the identity condition, was significant $(\beta=-0.085, \mathrm{SE}=0.012, t=-7.30$, related: $653 \mathrm{~ms}$ (SD 182) vs. identity: $595 \mathrm{~ms}$ (SD 143)). The second contrast, between the related and unrelated condition did not show a significant difference $(\beta=0.013$, SE $=0.011, t=1.18$, unrelated: $656 \mathrm{~ms}$ (SD 162)). Since the second contrast was not significant we cannot be certain that the identity condition differed not only from the related but also from the unrelated condition. To test this, the same model was run but with different contrast coding such that the unrelated condition was chosen as the baseline instead of the related condition. The new contrast between the identity condition and the unrelated condition was significant $(\beta=-0.098, \mathrm{SE}=0.010, t=-9.51)$.

In the plan condition, participants named the first picture as well. The average naming latency for these pictures was $734 \mathrm{~ms}(\mathrm{SD}=194)$. As expected, the kind of prime that was presented simultaneously with this picture did not affect these naming latencies as there was no relationship between the prime and picture. Including the main effect of condition did not improve model fit: $\chi^{2}(2)=2.46, p=0.29$. Finally, we found that naming latencies for these first (non-target) pictures were increased compared to naming latencies for the second (target) pictures: the full model was significantly better than the null model $\left(\chi^{2}(1)\right.$ $=86.64, p<0.001, \beta=-0.151, \mathrm{SE}=0.009, t=-17.15)$.

\subsection{Discussion}

The goal of Experiment 2 was to assess whether the pattern of results seen in Experiment 1 would be replicated in a within-participants design. Thus, the main question was whether the identity and associative priming effects would again be equal in strength in the no-plan and plan condition. For identity priming this was the case: Priming effects (when compared to the unrelated condition) of $78 \mathrm{~ms}$ and $61 \mathrm{~ms}$ were seen in the no-plan and in the plan condition, respectively. By contrast, for associative priming this was not the case: There was a significant priming effect of $16 \mathrm{~ms}$ in the no-plan condition, but there

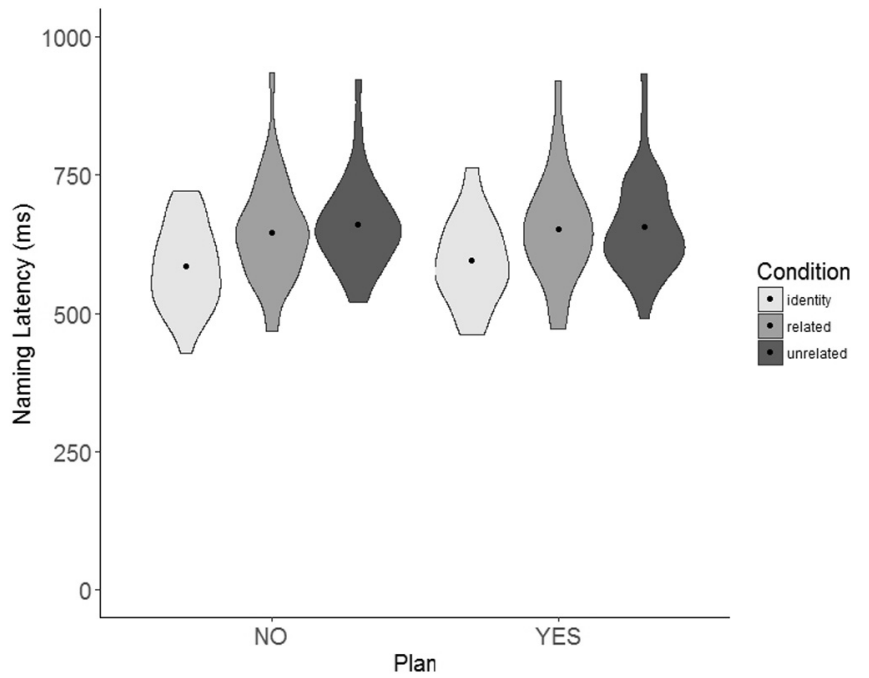

Fig. 3. Violin plot of mean naming latencies for target pictures following identity, associatively related and unrelated prime in the no-plan (NO) and plan (YES) condition. Dots indicate the condition mean.

was no priming $(3 \mathrm{~ms})$ in the plan condition.

The difference in the results obtained in the two experiments is not confined to the plan condition: As Figs. 2 and 3 show, the associative priming effects are reduced in Experiment 2, not only in the plan but also in the no-plan condition. By contrast, the identity priming effects in the two experiments are quite similar in both the plan and in the noplan condition. Statistical analyses comparing the two experiments confirmed this impression. For this analysis the data from Experiment 2 were split per condition. The no-plan condition was compared to the no-plan group and the plan condition to the plan group of Experiment 1. Whereas the conditions of Experiment 2 had half the number of trials as the plan and no-plan group, more than twice the number of participants was tested, resulting in similar trial counts. Linear mixed effects models were used with experiment and condition and their interaction as fixed effects and the same random structure as in Experiment 1. For the no-plan trials, removing the interaction resulted in worse model fit $\left(\chi^{2}(2)=6.72, p<0.05\right)$. Whereas the experiments showed similar effects for the difference between identity and associative primes ( $\beta=-0.020, \mathrm{SE}=0.019, t=-1.03$ ), the difference between associative and unrelated primes was different for the two experiments $(\beta=-0.066$, SE $=0.025, t=-2.60)$.

A similar result was obtained for the plan trials. Dropping the interaction from the model resulted in worse model fit $\left(\chi^{2}(2)=14.01\right.$, $p<0.001)$. This effect was driven by the difference between associatively related primes versus unrelated primes $(\beta=-0.074$, $\mathrm{SE}=0.023, t=-3.19$ ), not by the effect of identity primes relative to the related primes $(\beta=0.012, \mathrm{SE}=0.019, t=0.65)$. In sum, Experiment 2 revealed a modulated effect of associative priming. In the plan condition, associative priming disappeared. This was evident from both the within-participants analysis and the between-participants comparison to the plan group. In the no-plan condition, associative priming was present, as revealed by the within-participants analysis, but the effect was reduced compared to the no-plan group. Identity priming was present in both conditions.

Our findings are consistent with many reports in the literature that identity priming is stronger and more robust than other types of priming: Identity priming results in largest facilitation effects (Ferrand et al., 1994; Wheeldon \& Monsell, 1992) and has long-lasting effects of minutes, hours, or even weeks (Barry, Hirsh, Johnston, \& Williams, 2001; Cave, 1997; Jacoby \& Dallas, 1981). This is in contrast to other types of priming effects that are much more short-lived (Meyer, Schvaneveldt, \& Ruddy, 1972; Woltz, 1990). The difference in the strength and robustness of the priming effects is most likely related to 
differences in the underlying processing mechanisms. An identity prime is, by definition, the name of the upcoming target. Thus, priming can arise without access to any further lexical items. By contrast, the associative primes did not correspond to the target names, but were only related to them. For priming effects to arise, there has to be mediating processes (for instance automatic spreading or activation or strategic guessing) that lead to the targets.

A factor that could have contributed to the dissociation between the identity and associative priming effects in our study is that the identity primes reliably predicted the targets; i.e. they were always followed by the targets. By contrast, the associative primes were also used in the unrelated condition and therefore did not reliably predict the targets. This may have encouraged the strategic use of the primes more in the identity than in the associative condition (Bodner \& Masson, 2001; de Groot, 1984). In short, the associative primes were less directly related to the target than the identity primes and lent themselves less well to strategic processing. Hence, it is not surprising that the associative priming effect was more readily affected by the change of design than the identity priming effect.

Which differences between the experiments might have led to the variation in the size of the associative priming effects? Since the materials and the timing of the individual trials were the same in both experiments, the difference in the priming effects must be related to the overall structure of the experiment. As noted above, an important difference between the experiments was that in Experiment 1 participants could predict which objects they had to name: The participants in the plan-group named all objects, and the participants in the no-plan group named every other object and were prompted to do so by the alternation of red and green frames around the pictures. By contrast, for the participants of Experiment 2, the task was less predictable. Picture with red frames (non-targets) target pictures were always followed by pictures with green frames (targets) that had to be named; but pictures with green frames could be followed by further pictures with green frames or pictures with red frames (2 to 1 chance). Therefore participants could not prepare for naming or not-naming prior to picture onset, but had to do so upon presentation of pictures. This may be a costly process and may have interfered with the processing of the primes on both plan and no-plan trials.

In sum, in Experiment 2, the identity priming effect of Experiment 1 was replicated in the plan and no-plan condition, indicating that the participants processed the primes sufficiently to facilitate target naming regardless of whether or not they were planning an object name during prime presentation. The associative priming effect was overall reduced and absent in the plan-condition, indicating that participants were less likely to strategically use priming when predictability of both the current task and the identity of the next target was low.

\section{General discussion}

Language is primarily used in conversation. Several corpus studies have revealed that gaps between speaker turns can be remarkably short, suggesting that listening and utterance planning can overlap (Heldner \& Edlund, 2010; Stivers et al., 2009). Recent experimental studies also point to overlap between the two processes (Barthel et al., 2016; Bögels et al., 2015; Torreira, Bögels, \& Levinson, 2015). How do speakers combine the two tasks? From different lines of research, we know that both comprehension and production require attention (Huettig \& Janse, 2016; Jongman, Roelofs, \& Meyer, 2015; Shao et al., 2012). When two tasks that require attention are combined instead of being executed separately, performance deteriorates. This has been shown for both comprehension and production when combined with unrelated non-verbal tasks (Ferreira \& Pashler, 2002; Rodd et al., 2010). Fargier and Laganaro (2016) showed that performance in a word production task was worse when it was combined with a verbal than a nonverbal auditory discrimination task.

Fargier and Laganaro studied one direction of interference, namely the effect of listening on word planning. We tested the reverse direction of influence and asked whether word planning interfered with word comprehension. We investigated this issue in a novel paradigm using identity and associative priming effects as indices of comprehension. We tested the effects of spoken primes onto picture naming. We used a longer SOA ( $2 \mathrm{~s}$ ) between primes and picture targets than earlier studies (Alario et al., 2000; Ferrand et al., 1994; Perea \& Gotor, 1997). Most importantly, each spoken prime was combined with an unrelated nontarget picture. In the plan condition, participants named these pictures before naming the targets. In the no-plan condition they heard a recording of these picture names. Based on earlier results, we expected strong identity and somewhat weaker associative priming effects. The main question was whether or not the strength of these effects would differ between the plan and no-plan condition. In other words, we asked how the processing of the primes would be affected by the requirement to plan a word during prime presentation. We predicted strong priming effects in the no-plan condition, as attention could be focused on listening to the primes, and weaker priming effects in the plan condition, where participant had to focus their attention on word planning.

We observed a more complex pattern of results. In Experiment 1, using a between-participants design, identity and associative priming effects of similar magnitude were seen in the plan and no-plan groups. These results show that word planning per se does not interfere with word comprehension. However, our data revealed that the opposite does not hold, as comprehension interfered with production, consistent with previous picture-word interference studies (i.e., Dhooge \& Hartsuiker, 2012; Schriefers et al., 1990). Naming latencies were substantially longer for the non-target pictures, which were accompanied by spoken primes than for the target pictures which were not accompanied by such primes. However, this cost was modest since participants could still perform the task with only a slight delay when naming the first object while, importantly, keeping the benefit of priming for naming the second object.

In Experiment 2, using a within-participants design, the identity priming effect was replicated and again similar in strength in the plan and no-plan conditions. By contrast, the associative priming effect was weaker than in Experiment 1 and only significant in the no-plan but not in the plan condition. We already discussed this pattern above. The dissociation between the identity and associative priming effects points to different mechanisms underlying the two effects: Identity primes directly activated the target names and were likely to be recognized as target names and used strategically, whereas associative primes activated the targets indirectly and were less likely to be used strategically We proposed that the reduction of the associative priming effect in Experiment 2 compared to Experiment 1 was related to the difference in the designs of the two experiments and the predictability of the tasks. Participants in Experiment 1 knew before the onset of each picture whether or not it had to be named, whereas the participants of Experiment 2 could not predict their task with certainty. We propose that deciding on the task (naming or withhold a response) interfered with the processing of the primes. Evidently, this hypothesis needs to be substantiated in further research.

Taken together, the two studies demonstrated the robustness of the identity priming effects from spoken words to picture naming; this effect was obtained in both experiments with and without a concurrent word planning task. The associative priming effect was somewhat more fragile, but in Experiment 1 it was present both in the absence and presence of the word planning tasks. These findings demonstrate that people can efficiently divide their attention between speaking and listening such that their speech is only slightly delayed relative to a speaking-only condition, while the spoken input is processed thoroughly enough to prime upcoming pictures. These results are important for theories concerning the capacity demands of speaking and listening (Roelofs \& Piai, 2011; Ye \& Zhou, 2009). Further behavioral and neurobiological work is needed to elucidate exactly how people carry out such tasks, for instance which of the processing components involved in 
speech planning and listening can co-occur in time and which need to follow each other.

The results are also important for psycholinguistic theories of conversation. It is often argued that speakers align their linguistic representations, making dialogue an easier task than a monologue (Garrod \& Pickering, 2004). It has been shown that people indeed align by using the same words. Our results provide evidence that people benefit from such alignment at the lexical level as identity priming consistently supported speech planning. Associative priming has also been said to be instrumental in facilitating speech planning. Our work suggests that it might be less beneficial, especially when a speaker also has to decide ad hoc whether or not to speak. Having to make such decisions is, of course, a core feature of everyday conversation. Thus, one might speculate that associate priming might not play a major role in such contexts. However, further work is needed to assess the overall task demands of conversation and the ways speakers combine listening and speaking and are helped or hindered by the other person's input.

\section{Acknowledgements}

We thank Mirte Dikmans for running all experiments.Funding

This research did not receive any specific grant from funding agencies in the public, commercial, or not-for-profit sectors.Declaration of interest

The authors declare that they have no conflicts of interest with respect to their authorship or the publication of this article.

Appendix A. Associative relatedness scores prime - target combinations

\begin{tabular}{|c|c|c|c|c|c|c|}
\hline \multicolumn{3}{|l|}{ Target } & \multicolumn{4}{|l|}{ Prime } \\
\hline & & & \multicolumn{3}{|l|}{ Associated } & \multirow{2}{*}{$\begin{array}{l}\text { Unrelated } \\
\text { Name }\end{array}$} \\
\hline Name & Name Agr & Freq & Name & Assoc Score & Freq & \\
\hline appel & 100 & 24 & schil & 0.20 & 6 & spijker \\
\hline auto & 100 & 208 & garage & 0.31 & 15 & parasol \\
\hline baby & 92 & 79 & luier & 0.30 & 7 & paleis \\
\hline bed & 97 & 300 & laken & 0.26 & 2 & gif \\
\hline boek & 100 & 387 & pagina & 0.23 & 29 & kruimel \\
\hline boom & 100 & 137 & hout & 0.16 & 48 & veter \\
\hline bril & 100 & 36 & opticien & 0.29 & 1 & laken \\
\hline brood & 95 & 70 & kruimel & 0.28 & 4 & circus \\
\hline bus & 100 & 58 & halte & 0.27 & 6 & web \\
\hline clown & 100 & 5 & circus & 0.22 & 7 & hel \\
\hline dak & 100 & 57 & pannen & 0.21 & 45 & kluif \\
\hline deur & 100 & 376 & kier & 0.19 & 13 & ivoor \\
\hline dolfijn & 100 & 46 & flipper & 0.25 & 0 & kier \\
\hline doos & 100 & 39 & karton & 0.23 & 8 & lens \\
\hline duivel & 100 & 46 & hel & 0.18 & 22 & wortel \\
\hline fiets & 100 & 48 & dynamo & 0.30 & 1 & asbak \\
\hline fles & 100 & 112 & dop & 0.21 & 5 & invalide \\
\hline geweer & 90 & 46 & schot & 0.17 & 0 & regen \\
\hline gitaar & 100 & 6 & snaar & 0.29 & 5 & schil \\
\hline glas & 97 & 153 & scherf & 0.23 & 10 & flipper \\
\hline hamer & 97 & 11 & spijker & 0.24 & 10 & eiland \\
\hline heks & 97 & 19 & bezem & 0.25 & 4 & camping \\
\hline hond & 100 & 168 & kluif & 0.25 & 1 & dynamo \\
\hline huis & 100 & 630 & architect & 0.19 & 13 & pagina \\
\hline kaas & 100 & 53 & rasp & 0.16 & 1 & hengel \\
\hline kerk & 97 & 205 & orgel & 0.30 & 7 & scherf \\
\hline konijn & 100 & 23 & wortel & 0.15 & 37 & orgel \\
\hline koning & 97 & 100 & paleis & 0.23 & 32 & haard \\
\hline olifant & 100 & 10 & ivoor & 0.27 & 3 & station \\
\hline oog & 100 & 820 & lens & 0.18 & 6 & snaar \\
\hline paard & 100 & 158 & zadel & 0.29 & 12 & zender \\
\hline palmboom & 95 & 3 & eiland & 0.14 & 78 & chloor \\
\hline paraplu & 100 & 9 & regen & 0.13 & 55 & schot \\
\hline radio & 100 & 52 & zender & 0.22 & 12 & pannen \\
\hline ring & 100 & 34 & verloving & 0.16 & 4 & halte \\
\hline rolstoel & 92 & 6 & invalide & 0.20 & 3 & verloving \\
\hline schaap & 91 & 26 & wol & 0.25 & 10 & dop \\
\hline schoen & 100 & 68 & veter & 0.28 & 5 & opticien \\
\hline sigaret & 97 & 74 & asbak & 0.15 & 11 & landing \\
\hline slang & 100 & 27 & gif & 0.16 & 5 & wol \\
\hline spin & 100 & 9 & web & 0.29 & 4 & rasp \\
\hline tent & 100 & 27 & camping & 0.22 & 3 & zadel \\
\hline trein & 100 & 81 & station & 0.30 & 45 & luier \\
\hline
\end{tabular}




\begin{tabular}{|c|c|c|c|c|c|c|}
\hline vis & 97 & 73 & hengel & 0.14 & 4 & garage \\
\hline vliegtuig & 100 & 52 & landing & 0.27 & 9 & karton \\
\hline vuur & 94 & 104 & haard & 0.19 & 15 & bezem \\
\hline zon & 100 & 46 & parasol & 0.26 & 5 & architect \\
\hline zwembad & 97 & 15 & chloor & 0.29 & 1 & hout \\
\hline
\end{tabular}

Name Agr = name agreement of target picture.

Freq $=$ word frequency.

Assoc Score = associative relatedness score prime and target combination.

\section{Appendix B. Pilot study}

Participants

Twelve participants (11 female) were selected from the participant pool as reported for the three experiments in the main manuscript. The average age was 22.9 (range 20-29), and ten participants were right-handed.

\section{Procedure}

The same primes and target pictures were used as in the three experiments described in the main manuscript. However, the non-target picture was never presented. A trial started with the simultaneous presentation of a fixation cross in the middle of the screen, and the prime presented auditorily. The fixation cross stayed on the screen for $2 \mathrm{~s}$. Then the picture was presented for 250 ms. Finally, the fixation cross was presented for $1750 \mathrm{~ms}$.

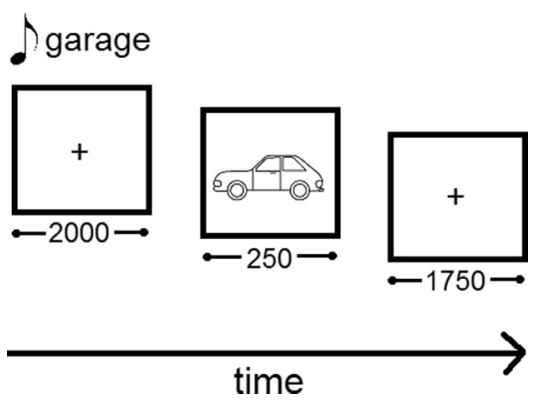

Fig. A1. Trial structure of pilot one. The example depicts a trail from the associatively related condition.

\section{Analysis}

The linear mixed effects model included condition (identity, related, and unrelated prime) and experiment half as fixed effects, and their interaction. Experiment half was included whether priming remains when the same prime-target pair is presented for a second time. As condition had three levels, contrast coding was chosen such that the related condition was compared to the identity and unrelated conditions. Variables were dropped that did not reliably contribute to model fit, models were compared using a likelihood ratio test. Participant, target, and prime were treated as random effects. For participant both intercept and random slopes were included for both fixed factors and their interaction, for target the interaction was excluded (only one observation per cell), for prime only the intercept was included.

Results

Naming errors occurred on average on $0.9 \%$ of all trials, and in an additional $0.4 \%$ of trials participants hesitated. No error analysis was performed due to the small number of mistakes. The best-fitting linear mixed effects model for correct naming latencies included main effects of condition and experiment half. Removing either condition or half significantly decreased model fit $\left(\chi^{2}(2)=34.16, p<0.001\right.$ and $\chi^{2}(1)=6.32$, $p<0.01$, respectively). Including the interaction did not improve model fit $\left(\chi^{2}(2)=5.53, p=0.06\right)$. For the best-fitting model, for condition both contrasts were significant. The first contrast, the related versus the identity condition ( $581 \mathrm{~ms}, \mathrm{SD}=193 \mathrm{vs} .509 \mathrm{~ms}, \mathrm{SD}=144)$, was significant $(\beta=-0.113, \mathrm{SE}=0.018, t=-6.35)$. The second contrast, between the related and unrelated condition (the latter with a mean of $652 \mathrm{~ms}$, $\mathrm{SD}=168)$ also showed a significant difference $(\beta=0.141, \mathrm{SE}=0.016, t=8.67)$. Experiment half also showed a significant effect $(\beta=-0.041$, $\mathrm{SE}=0.012, t=-3.29$ ). 


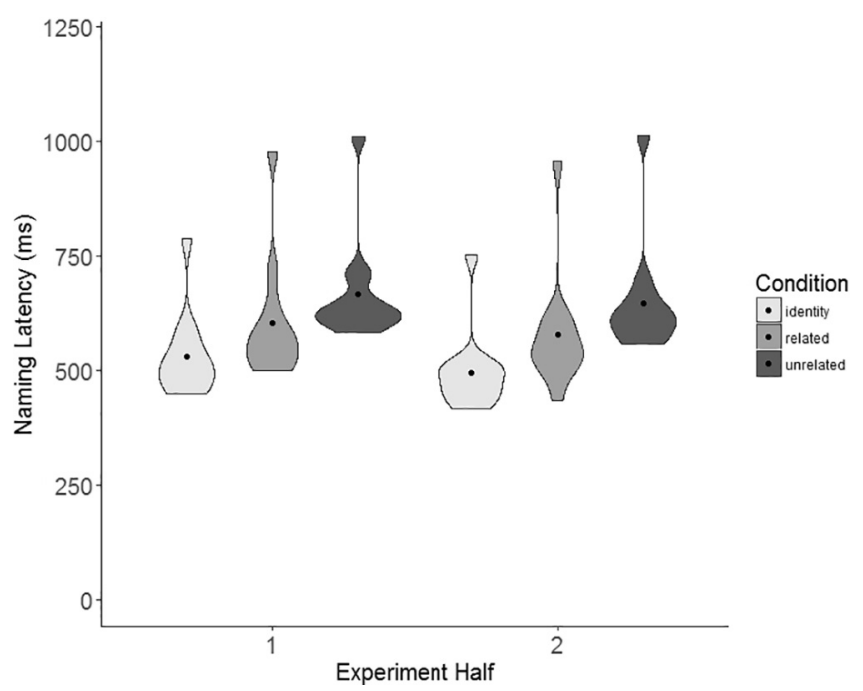

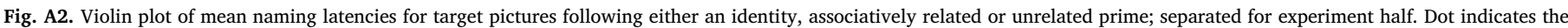
mean.

\section{References}

Abdel Rahman, R., \& Melinger, A. (2007). When bees hamper the production of honey: Lexical interference from associates in speech production. Journal of Experimental Psychology: Learning, Memory, and Cognition, 33(3), 604.

Alario, F.-X., Segui, J., \& Ferrand, L. (2000). Semantic and associative priming in picture naming. The Quarterly Journal of Experimental Psychology, 53(3), 741-764.

Baayen, R. H. (2008). Analyzing linguistic data. Vol. 505. Cambridge, UK: Cambridge University Press.

Baayen, R. H. (2011). languageR: Data sets and functions with "analyzing linguistic data: A practical introduction to statistics". R package version 1.4. Retrieved from http:// CRAN.R-project.org/package $=$ languageR.

Baayen, R. H., Piepenbrock, R., \& Gulikers, L. (1995). The CELEX lexical database. (CDROM). Philadelphia, PA: Linguistic Data Consortium, University of Pennsylvania.

Barr, D. J., Levy, R., Scheepers, C., \& Tily, H. J. (2013). Random effects structure for confirmatory hypothesis testing: Keep it maximal. Journal of Memory and Language, $68(3), 255-278$

Barry, C., Hirsh, K. W., Johnston, R. A., \& Williams, C. L. (2001). Age of acquisition, word frequency, and the locus of repetition priming of picture naming. Journal of Memory and Language, 44(3), 350-375.

Barthel, M., Sauppe, S., Levinson, S. C., \& Meyer, A. S. (2016). The timing of utterance planning in task-oriented dialogue: Evidence from a novel list-completion paradigm. Frontiers in Psychology, 7.

Bates, D., Maechler, M., \& Bolker, B. (2013). lme4: Linear mixed-effects models using S4 classes. R package version 0.999999-2. Retrieved from http://CRAN.R-project.org/ package $=$ lme 4 .

Bodner, G. E. \& Masson, M. E. J. (2001). Prime validity affects masked repetition priming: Evidence for an episodic resource account of priming. Journal of Memory and Language, 45(4), 616-647.

Boersma, P., \& Weenink, D. (2012). Praat: Doing phonetics by computer [computer program]. Version 5.3.32. Retrieved from http://www.praat.org/.

Bögels, S., Magyari, L., \& Levinson, S. C. (2015). Neural signatures of response planning occur midway through an incoming question in conversation. Scientific Reports, 5.

Brennan, S. E., \& Clark, H. H. (1996). Conceptual pacts and lexical choice in conversation. Journal of Experimental Psychology: Learning, Memory, and Cognition, 22(6), 1482.

Cave, C. B. (1997). Very long-lasting priming in picture naming. Psychological Science, 8(4), 322-325.

Collins, A. M., \& Loftus, E. F. (1975). A spreading-activation theory of semantic processing. Psychological Review, 82(6), 407.

Cook, A. E., \& Meyer, A. S. (2008). Capacity demands of phoneme selection in word production: New evidence from dual-task experiments. Journal of Experimental Psychology: Learning, Memory, and Cognition, 34(4), 886-899.

R Core Team (2012). R: A language and environment for statistical computing. Vienna, Austria: R Foundation for Statistical Computing. ISBN 3-900051-07-0, Retrieved from http://www.R-project.org/.

De Deyne, S., \& Storms, G. (2008). Word associations: Norms for 1,424 Dutch words in a continuous task. Behavior Research Methods, 40(1), 198-205.

de Groot, A. M. (1984). Primed lexical decision: Combined effects of the proportion of related prime-target pairs and the stimulus-onset asynchrony of prime and target. The Quarterly Journal of Experimental Psychology, 36(2), 253-280.

Dhooge, E., \& Hartsuiker, R. J. (2012). Lexical selection and verbal self-monitoring: Effects of lexicality, context, and time pressure in picture-word interference. Journal of Memory and Language, 66(1), 163-176.
Fargier, R., \& Laganaro, M. (2016). Neurophysiological modulations of non-verbal and verbal dual-tasks interference during word planning. PLOS ONE, 11(12), e0168358.

Ferrand, L., Grainger, J., \& Segui, J. (1994). A study of masked form priming in picture and word naming. Memory \& Cognition, 22(4), 431-441.

Ferreira, V. S., \& Pashler, H. (2002). Central bottleneck influences on the processing stages of word production. Journal of Experimental Psychology: Learning, Memory, and Cognition, 28(6), 1187-1199.

Garrod, S., \& Anderson, A. (1987). Saying what you mean in dialogue: A study in conceptual and semantic co-ordination. Cognition, 27(2), 181-218.

Garrod, S., \& Pickering, M. J. (2004). Why is conversation so easy? Trends in Cognitive Sciences, 8(1), 8-11.

Gomez, P., Perea, M., \& Ratcliff, R. (2013). A diffusion model account of masked versus unmasked priming: Are they qualitatively different? Journal of Experimental Psychology: Human Perception and Performance, 39(6), 1731.

Heldner, M., \& Edlund, J. (2010). Pauses, gaps and overlaps in conversations. Journal of Phonetics, 38(4), 555-568.

Huettig, F., \& Janse, E. (2016). Individual differences in working memory and processing speed predict anticipatory spoken language processing in the visual world. Language, Cognition and Neuroscience, 31(1), 80-93.

Indefrey, P., \& Levelt, W. J. M. (2004). The spatial and temporal signatures of word production components. Cognition, 92(1-2), 101-144.

Jacoby, L. L., \& Dallas, M. (1981). On the relationship between autobiographical memory and perceptual learning. Journal of Experimental Psychology: General, 110(3), 306.

Jongman, S. R., Roelofs, A., \& Meyer, A. S. (2015). Sustained attention in language production: An individual differences investigation. The Quarterly Journal of Experimental Psychology, 68(4), 710-730.

Keefe, D. E., \& Neely, J. H. (1990). Semantic priming in the pronunciation task: The role of prospective prime-generated expectancies. Memory \& Cognition, 18(3), 289-298.

Konopka, A. E., \& Meyer, A. S. (2014). Priming sentence planning. Cognitive Psychology, $73,1-40$.

Levinson, S. C., \& Torreira, F. (2015). Timing in turn-taking and its implications for processing models of language. Frontiers in Psychology, 6.

Meyer, D. E., Schvaneveldt, R. W., \& Ruddy, M. G. (1972). Activation of lexical memory. Paper presented at the meeting of the Psychonomic Society, St. Louis.

Moss, H. E., \& Marslen-Wilson, W. D. (1993). Access to word meanings during spoken language comprehension: Effects of sentential semantic context. Journal of Experimental Psychology: Learning, Memory, and Cognition, 19(6), 1254.

Navon, D., \& Miller, J. (1987). Role of outcome conflict in dual-task interference. Journal of Experimental Psychology: Human Perception and Performance, 13(3), 435.

Perea, M., \& Gotor, A. (1997). Associative and semantic priming effects occur at very short stimulus-onset asynchronies in lexical decision and naming. Cognition, 62(2), 223-240.

Pickering, M. J., \& Garrod, S. (2004). Toward a mechanistic psychology of dialogue. Behavioral and Brain Sciences, 27(02), 169-190.

Rodd, J. M., Johnsrude, I. S., \& Davis, M. H. (2010). The role of domain-general fronta systems in language comprehension: Evidence from dual-task interference and semantic ambiguity. Brain and Language, 115(3), 182-188.

Roelofs, A., \& Piai, V. (2011). Attention demands of spoken word planning: A review. Frontiers in Psychology, 2(307), 1-14.

Schriefers, H., Meyer, A. S., \& Levelt, W. J. M. (1990). Exploring the time course of lexical access in language production: Picture-word interference studies. Journal of Memory and Language, 29(1), 86-102.

Severens, E., Van Lommel, S., Ratinckx, E., \& Hartsuiker, R. J. (2005). Timed picture naming norms for 590 pictures in Dutch. Acta Psychologica, 119(2), 159-187. 
Shao, Z., Roelofs, A., \& Meyer, A. S. (2012). Sources of individual differences in the speed of naming objects and actions: The contribution of executive control. The Quarterly Journal of Experimental Psychology, 65(10), 1927-1944.

Shelton, J. R., \& Martin, R. C. (1992). How semantic is automatic semantic priming? Journal of Experimental Psychology: Learning, Memory, and Cognition, 18(6), 1191.

Sjerps, M. J., \& Meyer, A. S. (2015). Variation in dual-task performance reveals late initiation of speech planning in turn-taking. Cognition, 136, 304-324.

Stivers, T., Enfield, N. J., Brown, P., Englert, C., Hayashi, M., Heinemann, T., et al. (2009). Universals and cultural variation in turn-taking in conversation. Proceedings of the National Academy of Sciences, 106(26), 10587-10592.

Torreira, F., Bögels, S., \& Levinson, S. C. (2015). Breathing for answering: The time course of response planning in conversation. Frontiers in Psychology, 6(284).

Wheeldon, L. R., \& Monsell, S. (1992). The locus of repetition priming of spoken word production. The Quarterly Journal of Experimental Psychology, 44(4), 723-761.

Wickens, C. D. (2008). Multiple resources and mental workload. Human Factors, 50(3), $449-455$.

Woltz, D. J. (1990). Repetition of semantic comparisons: Temporary and persistent priming effects. Journal of Experimental Psychology: Learning, Memory, and Cognition, 16(3), 392.

Ye, Z., \& Zhou, X. (2009). Executive control in language processing. Neuroscience \& Biobehavioral Reviews, 33(8), 1168-1177. 